\title{
SYMMETRIES, LADDER OPERATORS AND QUANTUM INTEGRABLE SYSTEMS
}

\author{
A. P. FORDY \\ Department of Applied Mathematics, University of Leeds. Leeds LS2 9JT, UK \\ e-mail:allan@maths.leeds.ac.uk
}

(Received 10 December, 2003; accepted 27 October, 2004)

\begin{abstract}
We consider a class of deformations of Laplace-Beltrami operators on flat and constant curvature spaces, which possess a family of commuting operators. These are built as deformations of the symmetries of the underlying geometric space. In flat spaces it is also possible to extend some symmetries into ladder operators. In all cases it is possible to choose sub-classes which are super-integrable.
\end{abstract}

2000 Mathematics Subject Classification. 53C80, 81Q99.

1. Introduction. For an $n$-dimensional (pseudo-)Riemannian space, with local coordinates $x^{1}, \ldots, x^{n}$ and metric $g_{i j}$, the Laplace-Beltrami operator is defined by

$$
L_{b} f=\sum_{i, j=1}^{n} \frac{1}{\sqrt{g}} \frac{\partial}{\partial x^{j}}\left(\sqrt{g} g^{i j} \frac{\partial f}{\partial x^{i}}\right),
$$

where $g$ is the determinant of the matrix $g_{i j}$. When the space is either flat or constant curvature, it possesses the maximal group of isometries, which is of dimension $\frac{1}{2} n(n+1)$. The infinitesimal generators (Killing vectors) are just first order differential operators which commute with the Laplace-Beltrami operator (1). This is always the case, whenever the space has isometries, but in the case of flat and constant curvature spaces, $L_{b}$ is actually the second order Casimir function of the symmetry algebra (see [3]).

We may ask whether it is possible to deform this differential operator in such a way that it still possesses a number of first integrals. We are therefore led to consider the operator

$$
L f=L_{b} f+\sum_{i=1}^{n} A^{i} \frac{\partial f}{\partial x^{i}}+U f,
$$

where $A^{i}$ and $U$ are functions of the coordinates (the vector and scalar potentials). This poses the general question of determining those functions for which there exist $n$ or more commuting differential operators $I_{k}$. Since the leading order part of $I_{k}$ must commute with $L_{b}$ itself, this is not such a difficult task. The leading order part of $I_{k}$ defines a Killing tensor for the metric and, for flat and constant curvature spaces, these are just tensor products of Killing vectors $[\mathbf{1}, \mathbf{5}]$. In this short paper, we restrict attention to the case $n=2$, but the approach is easily applied to higher dimensions. In the case $n=2$, it is enough to find a single commuting operator for integrability, but it 
is also easy to construct super-integrable examples, possessing 2 independent operators which commute with $L$, but which are not, of course, in involution.

Recently, there has been much interest in such systems. In [4] they arise in the context of Krall-Sheffer polynomials [6]. In [2] some integrable Schrödinger operators with magnetic fields are found by using the factorisation method of Schrödinger. In [7] the authors carry out a "brute force" symmetry calculation to determine superintegrable systems in quantum mechanics.

In the current paper we consider deformations of first and second order symmetry operators, satisfying the ladder operator condition

$$
[L, S]=k S,
$$

where $k$ is any constant ( $k=0$ in the case of a symmetry). For a number of examples, there exist $S_{ \pm}$, corresponding to $k= \pm \tilde{k}$, so that the quantity $S_{+} S_{-}$is a symmetry (of either second or fourth order). This considerably reduces the complexity of the calculation.

Since we are not going to be involved in tensorial calculations, there is no need to use upper and lower index notation. All coordinates will carry lower indices in what follows.

2. Flat Metrics. The Euclidean group of motions in $n$ dimensions is the semidirect product of the group of $n$ (commuting) translations and the group $O(n)$ of rotations. In the flat coordinates for which the metric is given by

$$
d s^{2}=\sum_{i=1}^{n} d x_{i}^{2},
$$

the Euclidean algebra is generated by

$$
T_{i}=\partial_{i}, \quad \Omega_{i j}=x_{i} \partial_{j}-x_{j} \partial_{i}, \quad i<j,
$$

satisfying

$$
\left[T_{i}, T_{j}\right]=0, \quad\left[T_{i}, \Omega_{j k}\right]=\delta_{i j} T_{k}-\delta_{k i} T_{j}
$$

The quadratic Casimir of this algebra is just the Laplacian $\mathcal{C}_{2}=\sum_{i=1}^{n} T_{i}^{2}=\sum_{i=1}^{n} \partial_{i}^{2}$, which is (1) for this simple metric. In this paper we restrict ourselves to the case of $n=2$.

We consider the deformed operator

$$
L=\partial_{1}^{2}+\partial_{2}^{2}+A_{1} \partial_{1}+A_{2} \partial_{2}+U
$$

where $A_{i}, U$ are functions of the coordinates, and deform the two commuting translation operators $T_{i}$, requiring these to be ladder operators:

$$
S_{i}=\partial_{i}+\gamma_{i}, \quad\left[L, S_{i}\right]=k_{i} S_{i},
$$

where $\gamma_{i}$ are functions, to be determined, and $k_{i}$ constants. Since the leading order part of $S_{i}$ are symmetries of $L_{b}$, this imposes just 3 conditions (the coefficients of the first and zeroth order terms), the first 2 of which impose a constraint on the "electromagnetic 
field tensor" $B_{12}=\partial_{1} A_{2}-\partial_{2} A_{1}$. For $i=1$ this is

$$
\left.\begin{array}{r}
\partial_{1} \gamma_{1}=\frac{1}{2} k_{1}+\frac{1}{2} \partial_{1} A_{1} \\
\partial_{2} \gamma_{1}=\frac{1}{2} \partial_{1} A_{2}
\end{array}\right\} \Rightarrow \partial_{1} B_{12}=0
$$

with a similar result for $i=2$. Taking the 2 conditions together, leads to $B_{12}=b$, a constant (possibly zero). The final condition for each $i$, specifies the derivatives of $U$, whose integrability conditions give $k_{2}=-k_{1}$. In summary we have, up to gauge transformation, and putting $k_{1}=k$,

$$
\begin{aligned}
& A_{1}=-\frac{1}{2} b x_{2}, \quad A_{2}=\frac{1}{2} b x_{1}, \\
& \gamma_{1}=\frac{1}{2} k x_{1}+\frac{1}{4} b x_{2}, \quad \gamma_{2}=-\frac{1}{2} k x_{2}-\frac{1}{4} b x_{1}, \\
& U=\frac{1}{16}\left(b^{2}-4 k^{2}\right)\left(x_{1}^{2}+x_{2}^{2}\right)-\frac{1}{2} b k x_{1} x_{2} .
\end{aligned}
$$

As a result of the commutation relations (4), we have, for general $b$,

$$
\left[L, I_{1}\right]=0, \quad \text { where } I_{1}=S_{1} S_{2} .
$$

We also have that $S_{i}$ satisfy

$$
\left[S_{1}, S_{2}\right]=-\frac{1}{2} b
$$

2.1. Super-integrable case. When either $b=0$ or $k=0, L$ is rotationally invariant, meaning that $\left[L, \Omega_{12}\right]=0$, thus rendering the system super-integrable, with one second order and one first order commuting operator. In the case $k=0$ the former ladder operators actually commute with $L$, and

$$
L=S_{1}^{2}+S_{2}^{2}+\frac{1}{2} b \Omega_{12}
$$

In the case $b=0, L$ has the discrete symmetry $k \mapsto-k$, so instead of two ladder operators we have four! This is a reflection of the separability of this case. We have

$$
S_{i}^{ \pm}=\partial_{i} \pm \frac{1}{2} k x_{i}, \quad \text { satisfying } \quad\left[L, S_{i}^{ \pm}\right]= \pm k S_{i}^{ \pm}
$$

and

$$
\left[S_{i}^{+}, S_{j}^{-}\right]=-k \delta_{i j}, \quad\left[S_{i}^{+}, S_{j}^{+}\right]=\left[S_{i}^{-}, S_{j}^{-}\right]=0 .
$$

For each $i$ we have a 1-dimensional harmonic oscillator. It also follows from the commutation relations (5) that $\left[L, S_{i}^{+} S_{j}^{-}\right]=0$ for any choice of $i, j$. In particular

$$
L=S_{1}^{+} S_{1}^{-}+S_{2}^{+} S_{2}^{-}+k, \quad I_{1}=S_{1}^{+} S_{2}^{-}, \quad \Omega_{12}=\frac{1}{k}\left(S_{1}^{+} S_{2}^{-}-S_{2}^{+} S_{1}^{-}\right) .
$$

In this case we can gauge transform the potential function to a constant, after which $L$ can be transformed by a change of coordinates to a Krall-Sheffer "admissible 
operator". We have

$$
\left.\begin{array}{r}
L f=\lambda f \\
f=\varphi \tilde{f}
\end{array}\right\} \Rightarrow \tilde{L} \tilde{f}=\lambda \tilde{f}
$$

where

$$
\tilde{L}=\partial_{1}^{2}+\partial_{2}^{2}+\partial_{1} \varphi \partial_{1}+\partial_{2} \varphi \partial_{2}+\partial_{1}^{2} \varphi+\left(\partial_{1} \varphi\right)^{2}+\partial_{2}^{2} \varphi+\left(\partial_{2} \varphi\right)^{2}-\frac{1}{4} k^{2}\left(x_{1}^{2}+x_{2}^{2}\right) .
$$

Choosing $\varphi=\frac{1}{4} k\left(x_{1}^{2}+x_{2}^{2}\right)+\beta_{1} \log x_{1}+\beta_{2} \log x_{2}$ we find

$$
\tilde{L}=\partial_{1}^{2}+\partial_{2}^{2}+\left(k x_{1}+\frac{2 \beta_{1}}{x_{1}}\right) \partial_{1}+\left(k x_{2}+\frac{2 \beta_{2}}{x_{2}}\right) \partial_{2}+\left(\beta_{1}+\beta_{2}+1\right) k,
$$

whenever $\beta_{i}=0,1$. The choices $\left(\beta_{1}, \beta_{2}\right)=(0,0),(1,0),(1,1)$ correspond to KrallSheffer cases VII, VI, IV respectively (throughout this paper, the numbering is taken from [4]). This is discussed further in section 4.

2.2. Second order ladder operators. We now ask whether there exist nontrivial examples of (3) for which there exist second order ladder operators, which are extensions of some second order symmetries of the Laplace-Beltrami operator. Such an operator will be considered nontrivial if it is not the composition of a pair of first order ladder operators. We consider extensions of the operators $T_{i}^{2}$ :

$$
S_{i}=\partial_{i}^{2}+\sum_{j=1}^{2} \gamma_{i}^{j} \partial_{j}+\gamma_{i}^{0}, \quad\left[L, S_{i}\right]=k_{i} S_{i}
$$

where $\gamma_{i}^{j}, j=0,1,2$, are functions, to be determined, and $k_{i}$ constants. We now have 6 equations for the coefficient as a consequence of each of the ladder equations (7). This gives us 12 equations for 9 coefficients. The coefficients of quadratic derivatives give equations for $\gamma_{i}^{1}$ and $\gamma_{i}^{2}$, the integrability conditions of which give that $\partial_{1} \partial_{2} B_{12}=0$. Up to gauge transformation we may therefore write $A_{1}=a_{1}^{\prime}\left(x_{2}\right), A_{2}=a_{2}^{\prime}\left(x_{1}\right)$, after which

$$
\gamma_{1}^{1}=\frac{1}{2} k_{1} x_{1}+c_{1} x_{2}, \quad \gamma_{1}^{2}=a_{2}^{\prime}\left(x_{1}\right)-c_{1} x_{1} \quad \gamma_{2}^{1}=a_{1}^{\prime}\left(x_{2}\right)-c_{2} x_{2} \quad \gamma_{2}^{2}=\frac{1}{2} k_{2} x_{2}+c_{2} x_{1} .
$$

It is unnecessary to give a full classification here, so we simplify the formulae by setting $c_{1}=c_{2}=0, k_{2}=-k_{1}=-k$. The coefficients of the first order derivatives give equations for $\gamma_{i}^{0}$, whose integrability conditions give a formula for $U_{x_{1} x_{2}}$. This leads to

$$
\begin{aligned}
U\left(x_{1}, x_{2}\right)= & U_{1}\left(x_{1}\right)+U_{2}\left(x_{2}\right)-\frac{1}{2}\left(a_{1}\left(x_{2}\right) a_{2}^{\prime \prime}\left(x_{1}\right)+a_{2}\left(x_{1}\right) a_{1}^{\prime \prime}\left(x_{1}\right)\right) \\
& +\frac{1}{4} k\left(x_{1} a_{1}^{\prime}\left(x_{2}\right)+x_{2} a_{2}^{\prime}\left(x_{1}\right)+x_{1} x_{2} a_{2}^{\prime \prime}\left(x_{1}\right)\right),
\end{aligned}
$$

and

$$
x_{2} a_{1}^{\prime \prime \prime}\left(x_{2}\right)+4 a_{1}^{\prime \prime}\left(x_{2}\right)+x_{1} a_{2}^{\prime \prime \prime}\left(x_{1}\right)+4 a_{2}^{\prime \prime}\left(x_{1}\right)=0 .
$$


This equation is separable and leads (after removing some inessential constants) to

$$
a_{1}\left(x_{2}\right)=\frac{m_{1}}{x_{2}^{2}}, \quad a_{2}\left(x_{1}\right)=\frac{m_{2}}{x_{1}^{2}} \quad \text { with } \quad B_{12}=6\left(\frac{m_{2}}{x_{1}^{4}}-\frac{m_{1}}{x_{2}^{4}}\right)
$$

After this, we find $\gamma_{i}^{0}$ and fix $U_{1}\left(x_{1}\right)$ and $U_{2}\left(x_{2}\right)$ and find that $m_{2}=m_{1}=m$. The final result is as follows:

$$
\begin{aligned}
L & =\partial_{1}^{2}+\partial_{2}^{2}-\frac{2 m}{x_{2}^{3}} \partial_{1}-\frac{2 m}{x_{1}^{3}} \partial_{2}-\frac{\left(x_{1}^{2}+x_{2}^{2}\right)}{16 x_{1}^{4} x_{2}^{4}}\left(48 m^{2}+8 k m x_{1} x_{2}\left(x_{1}^{2}-x_{2}^{2}\right)+k^{2} x_{1}^{4} x_{2}^{4}\right) \\
S_{1} & =\partial_{1}^{2}+\frac{1}{2} k x_{1} \partial_{1}-\frac{2 m}{x_{1}^{3}} \partial_{2}+\frac{1}{16}\left(k^{2} x_{1}^{2}+4 k\left(1+\frac{2 m x_{2}}{x_{1}^{3}}\right)-\frac{48 m^{2}}{x_{1}^{4} x_{2}^{2}}\right) \\
S_{2} & =\partial_{2}^{2}-\frac{1}{2} k x_{2} \partial_{2}-\frac{2 m}{x_{2}^{3}} \partial_{1}+\frac{1}{16}\left(k^{2} x_{2}^{2}-4 k\left(1+\frac{2 m x_{1}}{x_{2}^{3}}\right)-\frac{48 m^{2}}{x_{1}^{2} x_{2}^{4}}\right)
\end{aligned}
$$

When $m=0$ these operators are just the squares of (5) and $L$ reduces to our previous example of a pair of harmonic oscillators. When $m \neq 0$, this is a new integrable example and the operators $S_{i}$ cannot be reduced. When $k=0$, then each of the operators $S_{i}$ actually commute with $L$, so the system is super-integrable, with second order integrals. When $k \neq 0$, the fourth order operator $S_{1} S_{2}$ is an integral.

3. Constant Curvature Metrics. In the case of constant curvature metrics we directly construct second order commuting operators. The non-zero curvature is an obstruction to the existence of first order ladder operators. If we were to treat constant curvature metrics in general, then it would be better to choose some canonical form, such as the "Riemannian" form, which explicitly shows it as conformally flat, with infinitesimal rotations as Killing vectors (see $[\mathbf{1}, \mathbf{8}]$ ). However, in this paper we just look at one example and in the form which arises in the context of Krall-Sheffer operators in [4] (case II).

The inverse metric takes the form

$$
g^{i j}=\left(\begin{array}{cc}
x^{2} & x y \\
x y & y^{2}-y
\end{array}\right)
$$

so the corresponding Laplace-Beltrami operator is

$$
L_{b}=x^{2} \partial_{x}^{2}+2 x y \partial_{x} \partial_{y}+\left(y^{2}-y\right) \partial_{y}^{2}+\frac{3}{2}\left(x \partial_{x}+y \partial_{y}\right)-\frac{1}{2} \partial_{y} .
$$

In these coordinates, the symmetry algebra is

$$
S_{1}=x \partial_{x}, \quad S_{2}=\sqrt{x y} \partial_{y}, \quad S_{3}=2 \sqrt{x y} \partial_{x}+\sqrt{\frac{y}{x}}(y-1) \partial_{y},
$$

satisfying

$$
\left[S_{1}, S_{2}\right]=\frac{1}{2} S_{2}, \quad\left[S_{1}, S_{3}\right]=-\frac{1}{2} S_{3}, \quad\left[S_{2}, S_{3}\right]=S_{1},
$$


with $L_{b}$ the Casimir:

$$
L_{b}=\frac{1}{2}\left(S_{2} S_{3}+S_{3} S_{2}+2 S_{1}^{2}\right)
$$

We consider the deformed operator

$$
L=L_{b}+A_{1} \partial_{x}+A_{2} \partial_{y}+U
$$

where $A_{i}, U$ are functions of the coordinates. For brevity, we restrict attention to the case when $A_{i}=0$. We first consider operators $L$ which commute with

$$
I_{1}=S_{1}^{2}+\gamma_{1}(x, y)
$$

The first condition is on $U$, as a result of an integrability condition on $\gamma_{1}$ :

$$
\gamma_{1 x}=\frac{1}{2}(1-y) U_{x}, \quad \gamma_{1 y}=\frac{1}{2} x U_{x} \Rightarrow \partial_{x}\left(x U_{x}+(y-1) U_{y}+U\right)=0 .
$$

This leads to

$$
\gamma_{1}=\frac{1}{2}\left((1-y) U+\gamma_{10}(y)\right)
$$

with

$$
x U_{x}+(y-1) U_{y}+U=\gamma_{10}^{\prime}(y)
$$

where $\gamma_{10}(y)$ is an arbitrary function. The general solution of this equation is

$$
U(x, y)=\frac{U_{1}\left(\frac{y-1}{x}\right)}{x}+\frac{U_{2}(y)}{y},
$$

where the substitution $U_{2}=\frac{y}{y-1} \gamma_{10}$ is used to simplify some later calculations. This is the most general potential for which (9) (with $A_{i}=0$ ) is integrable. Those who are familiar with separable systems in classical mechanics will recognise the feature of $U(x, y)$ depending upon 2 arbitrary functions of 1 variable. These variables are, indeed, the separation variables for this system. Defining $x_{1}=\frac{y-1}{x}, x_{2}=y$, gives

$$
\begin{aligned}
L & =\frac{-2}{x_{2}-1}\left(x_{1}^{2} \partial_{1}^{2}+x_{1} \partial_{1}-\frac{1}{2} x_{1} U_{1}\left(x_{1}\right)\right)+\frac{2 x_{2}}{x_{2}-1} \partial_{2}^{2}+\left(3 x_{2}-1\right) \partial_{2}+\frac{U_{2}\left(x_{2}\right)}{x_{2}}, \\
I_{1} & =x_{1}^{2} \partial_{1}^{2}+x_{1} \partial_{1}-\frac{1}{2} x_{1} U_{1}\left(x_{1}\right) .
\end{aligned}
$$

Demanding that there exists another first integral for $L$, places further constraints on $U_{i}$, which then fixes these arbitrary functions.

3.1. An additional integral which extends $S_{2}^{2}$. We next ask that the operator $L$ commutes with

$$
I_{2}=S_{2}^{2}+\gamma_{2}(x, y)
$$


The integrability condition for $\gamma_{2}$ is

$$
U_{1}^{\prime \prime}(z)+x^{3} U_{2}^{\prime \prime}(y)=0, \quad \text { where } z=\frac{y-1}{x},
$$

giving

$$
U_{1}(z)=c_{0} z+\frac{c_{1}}{z}+c_{2}, \quad U_{2}(y)=\frac{-c_{1}}{y-1}, \quad \gamma_{2}=-c_{0} \frac{y}{x}-c_{1} \frac{x}{y} .
$$

We thus have the super-integrable system with

$$
\begin{aligned}
& L=L_{b}+c_{0}\left(\frac{y-1}{x^{2}}\right)+\frac{c_{1}}{y}+\frac{c_{2}}{x}, \\
& I_{1}=x^{2} \partial_{x}^{2}+x \partial_{x}-c_{2}\left(\frac{y-1}{x}\right)-c_{0}\left(\frac{y-1}{x}\right)^{2}, \\
& I_{2}=x y \partial_{y}^{2}+\frac{1}{2} x \partial_{y}-\frac{c_{0} y}{x}-\frac{c_{1} x}{y} .
\end{aligned}
$$

3.2. An alternative additional integral which extends $S_{3}^{2}$. We next ask that the operator $L$ commutes with

$$
I_{3}=S_{3}^{2}+\gamma_{3}(x, y)
$$

instead of $I_{2}$. The integrability condition on $\gamma_{3}$ is

$$
U_{1}^{\prime \prime}(z)+\frac{6}{z} U_{1}^{\prime}(z)+\frac{6}{z^{2}} U_{1}(z)+x^{3} U_{2}^{\prime \prime}(y)=0, \quad \text { where } z=\frac{y-1}{x},
$$

giving

$$
U_{1}(z)=-\frac{c_{0}}{z}+\frac{c_{1}}{z^{2}}+\frac{c_{2}}{z^{3}}, \quad U_{2}(y)=\frac{c_{0}}{y-1}+d_{0}, \quad \text { and } \quad \gamma_{3}=\left(c_{0}-d_{0}\right) \frac{(y-1)^{2}}{x y}-\frac{c_{2} x y}{(y-1)^{2}} .
$$

We thus have the super-integrable system with

$$
\begin{aligned}
L= & L_{b}+\frac{c_{1} x}{(y-1)^{2}}+\frac{c_{2} x^{2}}{(y-1)^{3}}+\frac{d_{0}-c_{0}}{y} \\
I_{1}= & x^{2} \partial_{x}^{2}+x \partial_{x}-c_{1}\left(\frac{x}{y-1}\right)-c_{2}\left(\frac{x}{y-1}\right)^{2} \\
I_{3}= & 4 x y \partial_{x}^{2}+4 y(y-1) \partial_{x} \partial_{y}+\frac{y(y-1)^{2}}{x} \partial_{y}^{2}+(3 y-1) \partial_{x}+\frac{(y-1)^{2}}{2 x} \partial_{y} \\
& +\left(c_{0}-d_{0}\right) \frac{(y-1)^{2}}{x y}-\frac{c_{2} x y}{(y-1)^{2}}
\end{aligned}
$$

4. Eigenfunctions. In this section we discuss the eigenfunctions of some of the examples seen in sections 2 and 3 . We are particularly interested in the role played by the ladder operators and symmetries and the role of super-integrability. 


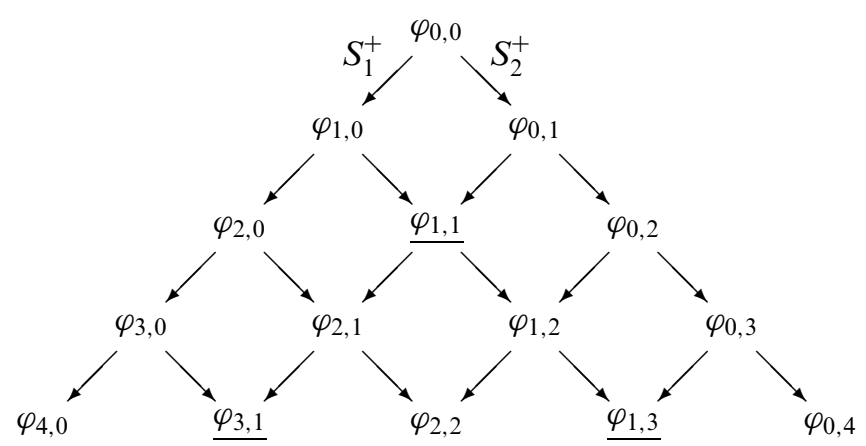

Figure 1. The triangular lattice. Underlining refers to polynomial eigenfunctions for the case $\beta_{i}=1$

4.1. Flat metrics. The easiest (non-trivial) case is when $b=0$ and $k \neq 0$. For $k=1$, the gauge transformed operator (6) (adjusting the additive constant term and removing the "tilde") and gauged ladder operators are

$$
\begin{aligned}
L & =\partial_{1}^{2}+\partial_{2}^{2}+\left(x_{1}+\frac{2 \beta_{1}}{x_{1}}\right) \partial_{1}+\left(x_{2}+\frac{2 \beta_{2}}{x_{2}}\right) \partial_{2}+\beta_{1}+\beta_{2}, \\
S_{i}^{ \pm} & =\partial_{i}+x_{i}+\frac{\epsilon_{i}^{ \pm} \beta_{i}}{x_{i}}, \quad \text { with } L=S_{1}^{+} S_{1}^{-}+S_{2}^{+} S_{2}^{-},
\end{aligned}
$$

where $\epsilon_{i}^{+}=1, \epsilon_{i}^{-}=0$ and $\beta_{i}=0,1$. As previously stated, $\left(\beta_{1}, \beta_{2}\right)=(0,0),(1,0),(1,1)$ correspond to Krall-Sheffer cases VII, VI, IV respectively. We start with the "ground state", corresponding to $S_{i}^{-} \varphi_{0,0}=0$ and hence $L \varphi_{0,0}=0$. We then build a triangular array of eigenfunctions (see Figure 1), defined by

$$
\varphi_{0,0}=x_{1}^{-\beta_{1}} x_{2}^{-\beta_{2}}, \quad \varphi_{m, n}=\left(S_{1}^{+}\right)^{m}\left(S_{2}^{+}\right)^{n} \varphi_{0,0} .
$$

This is a well defined array, since $\left[S_{1}^{+}, S_{2}^{+}\right]=0$. It follows from the commutation relations (with $k=1$ ) that $\varphi_{m, n}$ has eigenvalue $\lambda_{m, n}=m+n$. The precise form of these eigenfunctions depends upon the choice of $\beta_{i}$. However, we have the following general structure:

$$
\left.\begin{array}{r}
\varphi_{m, 0}=x_{2}^{-\beta_{2}}\left(S_{1}^{+}\right)^{m} x_{1}^{-\beta_{1}} \\
\varphi_{0, n}=x_{1}^{-\beta_{1}}\left(S_{2}^{+}\right)^{n} x_{2}^{-\beta_{2}} \\
\varphi_{m, n}=\left(\left(S_{1}^{+}\right)^{m} x_{1}^{-\beta_{1}}\right)\left(\left(S_{2}^{+}\right)^{n} x_{2}^{-\beta_{2}}\right)
\end{array}\right\} \Rightarrow \varphi_{m, n}=\frac{\varphi_{m, 0} \varphi_{0, n}}{\varphi_{0,0}} .
$$

Some of these eigenfunctions are polynomial. When $\beta_{1}=\beta_{2}=1$, then $\varphi_{1,1}=1$ and $\varphi_{m, 1}$ and $\varphi_{1, n}$ are respectively independent of $x_{2}$ and $x_{1}$ and therefore $\varphi_{m, n}=\varphi_{m, 1} \varphi_{1, n}$, for $m, n \geq 1$. When $m$ is odd, $\varphi_{m, 1}$ is a polynomial in $x_{1}^{2}$. Similarly, when $n$ is odd, $\varphi_{1, n}$ is a polynomial in $x_{2}^{2}$. This is indicated in Figure 1. by underlining. This suggests the change of coordinates $x=x_{1}^{2}, y=x_{2}^{2}$, after which

$$
\begin{aligned}
& L=2 x \partial_{x}^{2}+2 y \partial_{y}^{2}+(x+3) \partial_{x}+(y+3) \partial_{y}, \\
& I_{1}=2 x \partial_{x}^{2}+(x+3) \partial_{x}, \\
& I_{2}=x y\left(\partial_{x}-\partial_{y}\right)^{2}+(y-x)\left(\partial_{x}-\partial_{y}\right) .
\end{aligned}
$$


In these coordinates, $S_{i}^{ \pm}$have algebraic coefficients, but their squares have polynomial coefficients:

$$
\left(S_{1}^{+}\right)^{2}=4 x \partial_{x}^{2}+2(2 x+3) \partial_{x}+x+3, \quad\left(S_{1}^{-}\right)^{2}=4 x \partial_{x}^{2}+6 \partial_{x},
$$

and similarly for $S_{2}^{ \pm}$, so it is evident that the eigenfunctions of the indicated sub-lattice are themselves polynomial in $x$ and $y$.

4.2. Constant curvature metrics. We now consider the super-integrable case (12) and perform the gauge transformation $L \mapsto \tilde{L}=e^{-\varphi} L e^{\varphi}$ (and similarly for $I_{1}, I_{2}$ ), with

$$
\varphi=\alpha \frac{y-1}{2 x}+\frac{1}{2}(\beta+\gamma-1) \log x-\frac{1}{4}(2 \gamma+1) \log y
$$

where the constants are related to $c_{i}$ through

$$
c_{0}=\frac{1}{4} \alpha^{2}, \quad c_{1}=\frac{1}{4} \gamma^{2}+\frac{1}{2} \gamma+\frac{3}{16}, \quad c_{2}=\frac{1}{2} \alpha(2-\beta-\gamma),
$$

which leads to (after removing some additive constants and the "tilde")

$$
\begin{aligned}
& L=x^{2} \partial_{x}^{2}+2 x y \partial_{x} \partial_{y}+\left(y^{2}-y\right) \partial_{y}^{2}+(\beta x+\alpha) \partial_{x}+(\beta y+\gamma) \partial_{y} \\
& I_{1}=x^{2} \partial_{x}^{2}+((\beta+\gamma) x+\alpha(1-y)) \partial_{x}, \\
& I_{2}=x y \partial_{y}^{2}+(\alpha y-\gamma x) \partial_{y}
\end{aligned}
$$

which is just case II of the Krall-Sheffer system given in [4]. Since the operators $I_{1}, I_{2}$ commute with $L$, they correspond to degeneracy of the eigenvalues of $L$ by just shunting eigenfunctions around (whilst not changing the eigenvalue). We can mirror the construction of highest weight representations of Lie algebras by seeking eigenfunctions of $L$ which are in the kernel of $I_{1}$ and then using $I_{2}$ to build a sequence of eigenfunctions with the same eigenvalue. The kernel of $I_{1}$ (in the form (14)) consists of functions of $y$ only. We seek a sequence of polynomial eigenfunctions $P_{0 n}(y)=$ $y^{n}+$ "lower order terms", which must therefore be eigenfunctions of the reduced operator $L_{r}$ :

$$
L_{r} P_{0 n}=\lambda_{n} P_{0 n}, \quad L_{r}=y(y-1) \partial_{y}^{2}+(\beta y+\gamma) \partial_{y}, \quad \lambda_{n}=n(n+\beta-1),
$$

where the eigenvalue has been determined by comparing coefficients of $y^{n}$. It is straightforward to calculate these, the first few of which are

$$
\begin{aligned}
& P_{01}=y+\frac{\gamma}{\beta}, \quad P_{02}=y^{2}+2 \frac{\gamma-1}{\beta+2} y+\frac{(\gamma-1) \gamma}{(\beta+2)(\beta+1)} \\
& P_{03}=y^{3}+3 \frac{\gamma-2}{\beta+4} y^{2}+3 \frac{(\gamma-2)(\gamma-1)}{(\beta+4)(\beta+3)} y+\frac{(\gamma-2)(\gamma-1) \gamma}{(\beta+4)(\beta+3)(\beta+2)} .
\end{aligned}
$$

The sequence can be generated by the 3 point recursion relation:

$$
P_{0 n+1}=\left(y+a_{n}\right) P_{0 n}+b_{n} P_{0 n-1}
$$




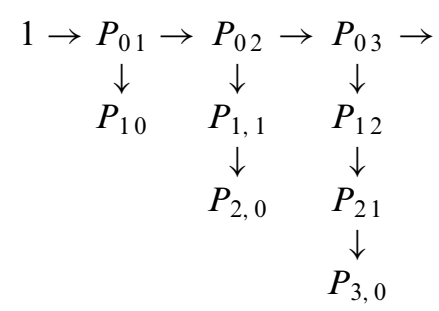

Figure 2. The lattice of polynomial eigenfunctions for the constant curvature system (14)

where

$$
\begin{aligned}
& a_{n}=\frac{(n+1)(\gamma-n)}{\beta+2 n}-\frac{n(\gamma-n+1)}{\beta+2 n-2}, \\
& b_{n}=-\frac{n(\gamma-n+1)}{2(\beta+2 n-2)}\left((n+1) \frac{\gamma-n}{\beta+2 n-1}-2 n \frac{\gamma-n+1}{\beta+2 n-2}+(n-1) \frac{\gamma-n+2}{\beta+2 n-3}\right) .
\end{aligned}
$$

We now define the sequence of companion polynomials $P_{m n-m}=c_{n m} x^{m} y^{n-m}+$ "lower order terms", where $c_{n m}$ are constant factors, determined by the recursion

$$
P_{m+1 n-m-1}=I_{2}\left(P_{m n-m}\right)-(n-m) \alpha P_{m n-m}, \quad I_{2}\left(P_{n 0}\right)=0 .
$$

The final equation follows from the fact that $P_{n 0}$ is a function of $x$ only. We just present a few of these:

$$
\begin{aligned}
& P_{10}=-\gamma\left(x+\frac{\alpha}{\beta}\right), \\
& P_{11}=-2(\gamma-1)\left(x y+\frac{1}{\beta+2}(\gamma x+\alpha y)+\frac{\alpha \gamma}{(\beta+2)(\beta+1)}\right), \\
& P_{20}=2(\gamma-1) \gamma\left(x^{2}+2 \frac{\alpha x}{\beta+2}+\frac{\alpha^{2}}{(\beta+2)(\beta+1)}\right) .
\end{aligned}
$$

The array of polynomial eigenfunctions is depicted in Figure 2.

5. Conclusions. In this paper it has been shown how to use the geometric symmetries of the Laplace-Beltrami operator of flat and constant curvature spaces to construct ladder operators and higher order symmetries of some extensions (2) of these operators. Here, we just presented 2 dimensional examples, for which the existence of just one independent commuting operator is enough to imply integrability (when considered as the Schrödinger operator of some quantum system). For each case considered, we presented sub-classes for which there existed an additional first integral (referred to as super-integrability). The existence of a commuting second order operator leads to the usual separable (by change of coordinates) systems. This can be seen explicitly in (11). However, we also presented a case (8) for which the commuting operator is fourth order, possessing second order ladder operators. The formula (10) shows that the existence of just one commuting operator can lead to a potential depending upon arbitrary functions. Demanding the existence of an additional 
commuting operator (super-integrability) restricts these functions to depending upon a few arbitrary constants (see (12) and (13)).

The imposition of super-integrability is so powerful that it enables us to construct eigenfunctions by elementary means. The existence of so many symmetries means that the eigenvalues are highly degenerate. As already noted in [4] the Krall-Sheffer operators fall into this category. In these cases some of the properties of "admissibility" of the operator follow directly from the super-integrability.

Whilst this paper has only been concerned with 2 dimensional systems the ideas were initially discussed in the context of general dimension, since it is an easy matter to repeat many of these calculations in this general context. It is also not necessary to restrict to constant curvature, since the only requirement is for a high degree of symmetry. Some of these ideas will be explored in further papers.

\section{REFERENCES}

1. L. P. Eisenhart, Riemannian geometry (Princeton University Press, 1926).

2. E. V. Ferapontov and A. P. Veselov, Integrable Schrödinger operators with magnetic fields: Factorization method on curved surfaces, J. Math. Phys. 42 (2001), 590-607. 1974).

3. R. Gilmore, Lie groups, Lie algebras and some of their applications (Wiley, New York,

4. J. Harnad, L. Vinet, O. Yermolayeva and A. Zhedanov, Two-dimensional Krall-Sheffer polynomials and integrable systems, J. Phys. A 34 (2001), 10619-10625.

5. G. H. Katzin and I. Levine, Quadratic first integrals of the geodesics in spaces of constant curvature, Tensor 10 (1965), 97-104.

6. H. L. Krall and I. M. Sheffer, Orthogonal polynomials in two variables, Ann. Mat. Pure Appl. Ser. 476 (1997), 325-376.

7. M. B. Sheftel, P. Tempesta and P. Winternitz, Superintegrable systems in quantum mechanics and classical Lie theory. J. Math. Phys. 42 (2001), 659-673.

8. M. Spivak, Differential Geometry, Vols I-V (Publish or Perish, Boston, 1970). 
